\title{
INFORMATION FLOW IN LOGICS IN THE VICINITY OF BB
}

\author{
ANDREW TEDDER \\ Institute of Computer Science \\ The Czech Academy of Sciences
}

\begin{abstract}
Situation theory in general, and channel theory in particular, have been used to provide motivational accounts of the ternary relation semantics of relevant, substructural, and various non-classical logics. Among the constraints imposed by channel-theory, one must posit a certain existence criterion for situations which result from the composites of multiple channels (this is used in modeling information flow). In logics obeying a certain associativity condition, it is relatively straightforward to show that a certain such condition is met, but the problem is trickier in non-associative logics. Following Tedder (2017), where it was shown that the conjunction-conditional fragment of the logic $\mathbf{B}$ admits the existence of composite channels, I present a generalised version of the previous argument, appropriate to logics with disjunction, using the neighbourhood ternary relation semantic framework. I close by suggesting that the logic $\mathbf{B} \mathbf{B}_{(\wedge \mathrm{I})}^{+}$, which falls in between Lavers' system $\mathbf{B B}^{+}$and the standard 'minimal' relevant logic $\mathbf{B}^{+}$, satisfies the conditions for the general argument to go through.
\end{abstract}

\section{INTRODUCTION}

The tradition of situation semantics, coming out of work of Barwise and Perry in the early 80s, most famously [7], has long provided the means to interpret and motivate the ternary relation semantics for relevant and substructural logics. This connection, noted even in 1985 by Barwise and Perry [7, p.xvii], was discussed by relevant logicians in [1, p.333]. Since then, something like the situation-theoretic reading has been widely adopted for FDE, where the major departure from classical logic is in permitting both incomplete theories and non-trivially inconsistent theories. ${ }^{1}$ Extensions of the situation-theoretic picture with conditional information have been used to motivate/interpret the distinctive truth conditions for conditional formulas used in the ternary relation semantics. Most notable is the tradition

E-mail address: ajtedder at@gmail.com.

I gratefully acknowledge the GaČR grant no. 18-19162Y (NOCLID) for funding. For helpful discussions, I'd like to thank Shay Allen Logan, Teresa Kouri Kissel, and Grace Paterson, as well as an audience at the Ruhr-Universität Bochum. Finally, I'd like to thank the AKH in Vienna for providing a good writing environment and Luna, who is responsible for my being there.

${ }^{1}$ Indeed, this interpretation of FDE was presaged before the advent of situation semantics in Belnap's [10].

Australasian Journal of Logic (18:1) 2021, Article no. 1 
of channel theory, introduced by Barwise [4], which seeks to explain conditional information in terms of channels. These are objects (perhaps more situations, perhaps not) which form connections between pairs of situations, allowing information to flow from one to the other. Barwise along with a number of co-authors, and many in the relevant logic tradition, have sought to adapt channel theory or some other kind of situation-theoretic account of conditional information (such as Israel and Perry's theory) to the relational semantics of relevant and substructural logics - for examples, see [6, 22, 23, 29].

Among the features of such accounts of conditional information are various constraints on channels, or situations supporting conditional information. For my purposes here, the most salient property concerns the serial composition of conditionalsupporting channels/situations. Barwise [4] introduces the constraint that for any two channels, there exists a channel composing them, which must satisfy certain properties. Tedder [39] proved that the weak relevant logic $\mathbf{B}^{\wedge}$ is complete with respect to those of its ternary relation models in which such composites exist, using some machinery developed by [2], but the method used there does not extend to $\mathbf{B}^{+}$, because of the interaction between disjunction and the conditional.

There are two aims of this paper. The first is to provide a more general version of the argument of [39] using the Neighbourhood Ternary relation (NTR) semantics developed by Sylvan (née Routley) and Meyer [32,33]. This will provide a collection of sufficient conditions for a logic to be complete with respect to the class of its NTR models satisfying a strong composites-existence property. For this purpose, I'll first present those elements of the NTR semantics which are needed for this purpose (alongside the usual TR semantics for comparison) and briefly discuss some of the existing work on how to understand composition (and a related principle) in the TR semantic framework. In so doing, I'll argue that we should not, pace Restall [29], require frames to satisfy the defining condition for the axiom form of suffixing. ${ }^{2}$ Then, I'll discuss a logic $\mathbf{B B}_{(\wedge \mathrm{I})^{\prime}}^{+}$extending $\mathbf{B}^{\wedge}$ by slightly weaker disjunction principles than $\mathbf{B}^{+}$, and present some evidence that it satisfies the sufficient conditions for completeness with respect to its NTR models in which channel composites exist. The upshot will be that there are reasons to think that logics in the vicinity of BB can be endowed with NTR frames appropriate to model the interaction between channels and situations, at least as concerns serial composition.

\section{TERnARy RElation SEMANTiCs: Two DifFEREnt Ones}

2.1. Languages. I'll be working throughout in a small handful of propositional languages, since I'll be discussing properties of logics which are sensitive to language extensions. To that end, the following definition is general, but basically standard.

- $\mathbb{P}$ is a set of atomic sentence letters - these are represented by lower-case letters of the Latin alphabet.

${ }^{2}$ Similar reasoning applies to the constraint defining the axiom form of prefixing, though I won't dwell on that.

Australasian Journal of Logic (18:1) 2020, Article no. 1 
- The basic set of connectives $C$ has the elements $\wedge, \rightarrow$, each of arity 2. $C_{[V]}$ extends this by the binary connective $V$, and similar naming conventions apply for $\circ, \leftarrow, \neg$ (arity 2, 2, 2, and 1, respectively) where they come up.

$\mathcal{L}_{0}$ is the set constructed from $\mathbb{P}$ and $C$ in the usual manner, and $\mathcal{L}_{[\mathrm{V}]}$ substitutes $C_{[V]}$ for $C$, and similarly for other sets of connectives. When context will distinguish, or where the differences between these various languages don't matter, I'll use $\mathcal{L}$ as a variable over these. Throughout, I'll use $\&$ and $\Rightarrow$ as metalinguistic connnectives - these will behave as classical conjunction and material implication - and furthermore all uses of quantifiers are metalinguistic. Universally quantified metalanguage expressions will usually just be written with bald variables. Finally, $\wedge, \vee$ bind more strongly than $\rightarrow$.

2.2. Ternary Relation (TR) Semantics. Introduced by Sylvan (née Routley) and Meyer in [31], following on earlier work by Sylvan and Plumwood (née Routley) [36] and further developed in [35, 12] among other places, the ternary relation (or "Routley-Meyer") semantics operates on frames of the following type (I omit the * used in interpreting negation):

where these elements are:.

$$
\mathfrak{F}=\langle W, N, R\rangle
$$

- $\varnothing \neq N \subseteq W$

- $R \subseteq W^{3}$

In addition to these, an order $\leq \subseteq W^{2}$ is defined:

$$
\alpha \leq \beta:=\exists \gamma \in N(R \gamma \alpha \beta)
$$

and frame elements are subject to the following constraints:

- $\langle W, \leq\rangle$ is a partially ordered set

- $N \in \wp(W)^{\uparrow}=\{X \subseteq W \mid(\alpha \in X \& \alpha \leq \beta) \Rightarrow \beta \in X\}$

- $\left(R \alpha \beta \gamma \& \alpha^{\prime} \leq \alpha \& \beta^{\prime} \leq \beta \& \gamma \leq \gamma^{\prime}\right) \Rightarrow R \alpha^{\prime} \beta^{\prime} \gamma^{\prime}$

Intuitively a frame includes a set of situations, a some of which, $N$, support all the logical truths, and a ternary $R$ which interprets the implication - I'll get into this shortly. The defined order $\leq$ expresses containment between situations - i.e. whenever $\alpha \leq \beta, \beta$ will support any proposition supported by $\alpha$, similarly to the heredity constraint in the frame semantics for intuitionist logic. The elements of $\wp(W)^{\uparrow}$, upward closed sets of situations, model propositions.

Given a frame $\mathfrak{F}$, the full complex algebra on $\mathfrak{F}$ is defined:

$$
\left\langle\wp(W)^{\uparrow}, N, \cap, \cup, \rightarrow, \leftarrow, \circ\right\rangle
$$

where for all $X, Y \subseteq W$ :

- $X \rightarrow Y=\{\alpha \in W \mid \forall \beta, \gamma \in W((R \alpha \beta \gamma \& \beta \in X) \Rightarrow \gamma \in Y)\}$

- $X \leftarrow Y=\{\alpha \in W \mid \forall \beta, \gamma \in W((R \beta \alpha \gamma \& \beta \in Y) \Rightarrow \gamma \in X)\}$

- $X \circ Y=\{\alpha \in W \mid \exists \beta, \gamma \in W(R \beta \gamma \alpha \& \beta \in X \& \gamma \in Y)\}$

The following results are standard:

Proposition 2.1. When $X, Y \in \wp(W)^{\uparrow}$, so is $X \otimes Y$ for $\otimes \in\{\rightarrow, \leftarrow, 0\}$.

Australasian Journal of Logic (18:1) 2020, Article no. 1 
Proposition 2.2. For $X, Y \in \wp(W)^{\uparrow}, N \subseteq X \rightarrow Y \Longleftrightarrow X \subseteq Y$

A model $M$ on a frame $\mathfrak{F}$ is a valuation function $V: \mathbb{P} \longrightarrow \wp(W)^{\uparrow}-$ when $M$ is on frame $\mathfrak{F}$, elements of $\mathfrak{F}$ are picked out by $W_{M}, N_{M}, R_{M}$ as needed (where context makes this obvious, the convention of explicitly noting the model may be dropped). This is extended to a valuation on the full language $\llbracket \cdot \rrbracket_{M}: \mathcal{L} \longrightarrow \wp(W)^{\uparrow}$ as follows:

- $\llbracket p \rrbracket_{M}=V(p)$ for $p \in \mathbb{P}$

- $\llbracket A \wedge B \rrbracket_{M}=\llbracket A \rrbracket_{M} \cap \llbracket B \rrbracket_{M}$

- $\llbracket A \vee B \rrbracket_{M}=\llbracket A \rrbracket_{M} \cup \llbracket B \rrbracket_{M}$

- $\llbracket A \otimes B \rrbracket_{M}=\llbracket A \rrbracket_{M} \otimes \llbracket B \rrbracket_{M}$ for $\otimes \in\{\rightarrow, \leftarrow, \circ\}$

$\alpha \in \llbracket A \rrbracket_{M}$ is often written as $M, \alpha \Vdash A$, or some variation thereon. Finally, $A$ is true-in- $M$ if and only if $N_{M} \subseteq \llbracket A \rrbracket_{M} . A$ is furthermore valid-in-F iff true-in- $M$ for every $M$ on $\mathfrak{F}$, and where $\mathcal{F}$ is a set of frames, $A$ is valid-in- $\mathcal{F}$ iff it is valid in all the frames in $\mathcal{F}$. These are written $\vDash_{+} A$ where + is the name of a model, frame, or set of frames. ${ }^{3}$

2.3. Neighbourhood Ternary Relation (NTR) Semantics. The Neighbourhood TR semantics can be understood as capitalising on the central feature that $N \subseteq X \rightarrow$ $Y \Longleftrightarrow X \subseteq Y$, while generalising the TR framework. Some of the complexity of the TR semantics arises from treating of this feature via situations, as it were. By generalising just a bit, it can be captured more directly and simply. Sylvan and Meyer introduced this framework in $[32,33]$ (a particularisation of yet more general semantics [34]) in order to provide a general account of implication connectives. I'll consider the framework as also incorporating the connectives $\wedge, \vee$ (in their 'normal' varieties as per [32]). ${ }^{4}$ Here an NTR frame is a structure:

$$
\mathfrak{F}=\langle W, N, R, \text { Prop }\rangle
$$

where $W, N$ are as before, and furthermore:

- Prop $\subseteq \wp(W)$

- $R \subseteq W \times \wp(W) \times \wp(W)$

satisfying the constraints:

(C0) Prop is closed w.r.t. $\cap, \cup$, and $\rightarrow$, where

$$
X \rightarrow Y=\{\alpha \in W \mid R \alpha X Y\}
$$

(C1) $N \subseteq X \rightarrow Y \Longleftrightarrow X \subseteq Y$, for all $X, Y \in$ Prop

(C2) $X=X^{\prime}, Y=Y^{\prime}$ only if $X \rightarrow Y=X^{\prime} \Rightarrow Y^{\prime}$

\footnotetext{
${ }^{3}$ There are a number of options available for defining relations of consequence (Tarski, Scott, or otherwise) of relevant logics, but some are better than others, depending on the purpose - see [1, p.169] for some discussion. For the sake of simplicity, I'll deal here just with theoremhood, and leave considerations of the correct notion of relevant logical consequence to the side.

${ }^{4}$ Goble [17] further developed (a variant on) the NTR framework, proving adequacy and decidability results for a number of systems similar to those discussed in this paper.
}

Australasian Journal of Logic (18:1) 2020, Article no. 1 
The inclusion of Prop is not strictly necessary, and involves a bit of additional complexity in the definitions - having said that, it makes the canonical model construction part of the completeness argument simpler, so I'll keep it.

Here, (C1) is the only substantial constraint - reflecting this basic commitment concerning conditionals encoded in Prop 2.2. In fact, this is closely connected to the justification of the basic properties of conditionals given by Sylvan and Meyer [32] (following a comment from Suszko).

A model $M$ on an NTR frame is a $V: \mathbb{P} \longrightarrow \wp(W)$, with $\llbracket \cdot \rrbracket_{M}$ being defined as before, excepting that now:

$$
\llbracket A \rightarrow B \rrbracket_{M}=\llbracket A \rrbracket_{M} \rightarrow \llbracket B \rrbracket_{M}=\left\{\alpha \mid R \alpha \llbracket A \rrbracket_{M} \llbracket B \rrbracket_{M}\right\}
$$

The definition of the various $\vDash^{\prime}$ s are as before. The basic NTR logic F is axiomatisable as follows - I shall use an " $\mathrm{r}$ " to distinguish the rules from the axioms, as similar names will recur again in the next section. ${ }^{5}$ The $\Rightarrow$ used in the statement of the rules should be understood to express the fact that these are rules of proof -i.e. $A_{1}, \ldots, A_{n} \Rightarrow B$ reads "from derivations of $A_{1}, \ldots, A_{n}$, one may derive $B$. "

(Id) $A \rightarrow A$

$(\wedge \mathrm{E}) A \wedge B \rightarrow A, A \wedge B \rightarrow B$

( $\vee \mathrm{I}) A \rightarrow A \vee B, B \rightarrow A \vee B$

(Dist) $A \wedge(B \vee C) \rightarrow(A \wedge B) \vee(A \wedge C)$

(rMP) $A \rightarrow B, A \Rightarrow B$

(rAdj) $A, B \Rightarrow A \wedge B$

$(\mathrm{r} \wedge \mathrm{I}) A \rightarrow B, A \rightarrow C \Rightarrow A \rightarrow B \wedge C$

$(\mathrm{r} \vee \mathrm{E}) A \rightarrow C, B \rightarrow C \Rightarrow A \vee B \rightarrow C$

(rWB) $A \rightarrow B, B \rightarrow C \Rightarrow A \rightarrow C$

(rCong) $A^{\prime} \leftrightarrow A, B^{\prime} \leftrightarrow B \Rightarrow(A \rightarrow B) \rightarrow\left(A^{\prime} \rightarrow B^{\prime}\right)$

Given a logic $\mathbf{L}$ defined by some axiomatic presentation, let $\vdash_{\mathbf{L}}$ express theoremhood of that logic as usual - i.e. every axiom of $\mathbf{L}$ is a theorem of $\mathbf{L}$, and any formula obtained from theorems of $\mathbf{L}$ by the use of rules of $\mathbf{L}$ is a theorem of $\mathbf{L}$.

2.4. Some NTR Frame Constraints. A key part of this semantics, as in all frame semantics, is the association between valid axioms/rules, and frame constraints, closely tying logics to certain NTR frames (for our purposes, the one which most matters is that of the canonical model).

In the NTR semantics, this is pretty trivial, at least as concerns the usual axiomatic extensions of the basic relevant logics. One need only rewrite the formula you want in the language of the frame, and $\mathbf{F}$ will be extended to obtain the logic

\footnotetext{
${ }^{5}$ The name $\mathbf{F}$ is used in [32]. This system is called Min by Goble [17], and a closely related system is discussed under the name "B" in [9, p. 93].

Australasian Journal of Logic (18:1) 2020, Article no. 1
} 
of those frames by the addition of the axiom matching the condition. Logics resulting from $\mathbf{F}$ by extensions by the following axioms and rules are important for our purposes. $^{6}$

(rB) $A \rightarrow B \Rightarrow(C \rightarrow A) \rightarrow(C \rightarrow B)$

$\left(\mathrm{rB}^{\prime}\right) A \rightarrow B \Rightarrow(B \rightarrow C) \rightarrow(A \rightarrow C)$

$(\wedge \mathrm{I})(A \rightarrow B) \wedge(A \rightarrow C) \rightarrow(A \rightarrow B \wedge C)$

$(\vee \mathrm{E})(A \rightarrow C) \wedge(B \rightarrow C) \rightarrow(A \vee B \rightarrow C)$

(WB) $(A \rightarrow B) \wedge(B \rightarrow C) \rightarrow(A \rightarrow C)$

(B) $(A \rightarrow B) \rightarrow((C \rightarrow A) \rightarrow(C \rightarrow B))$

$\left(\mathrm{B}^{\prime}\right)(A \rightarrow B) \rightarrow((B \rightarrow C) \rightarrow(A \rightarrow C))$

In keeping with the triviality claim, here are two examples of NTR frame constraints defining these rules/axioms (the others follow the same pattern):

$(\mathrm{rB}) X \subseteq Y \Rightarrow Z \rightarrow X \subseteq Z \rightarrow Y$

$(\wedge \mathrm{I})(X \rightarrow Y) \cap(X \rightarrow Z) \subseteq X \rightarrow(Y \cap Z)$

The basic idea here is that, as generally holds in relevant logics and their NTR models, a conditional formula $A \rightarrow B$ is valid just in case every element of an NTR model will satisfy $A$ (or "is a member of $A$ 's proposition") only if it satisfies $B$. That is, $A \rightarrow B$ will be valid iff for every $M, \llbracket A \rrbracket_{M} \subseteq \llbracket B \rrbracket_{M}$, as set out in (C1). So in order to guarantee that $(\wedge \mathrm{I})$ is valid, we can just ensure that whenever $\alpha \in \llbracket(A \rightarrow$ $B) \wedge(A \rightarrow C) \rrbracket_{M}$ then $\alpha \in \llbracket A \rightarrow B \wedge C \rrbracket_{M}$, which is what the homophonic NTR frame constraint delivers. In the case of rules, the only difference is that we have to consider (meta-level) implications between inclusions to model rules allowing us to infer implication formulas from other implication formulas. So the NTR frame constraint for $(\mathrm{rB})$ just recapitulates that rule at the level of implications between inclusion statements between propositions.

The logic resulting from adding the first four principles listed above, i.e. (rB)$(V E)$, to $\mathbf{F}$ is $\mathbf{B}$, the logic of all TR frames and the 'basic affixing system'. ${ }^{7}$ The addition of only the first two is BB, a logic motivated by Peter Lavers [20,21] as a particularly nice extension of FDE better capturing tautological entailments, as originally presented by Anderson and Belnap. I'll write the name of a logic with a subscripted name of one of these axioms in order to name a system of interest - for my purposes, the most important one here is $\mathbf{B} \mathbf{B}_{(\wedge I)}$.

Proposition 2.3. If $\mathbf{L}$ extends $\mathbf{B B}$ in the language $\{\rightarrow, \wedge\}$ and has $(\wedge \mathrm{I})$, then for any NTR frame for $\mathbf{L}$, and $\left\{X_{i}\right\}_{i \in I},\left\{Y_{i}\right\}_{i \in I} \subseteq \operatorname{Prop}$ (for finite $I$ ):

$$
\bigcap_{i \in I}\left(X_{i} \rightarrow Y_{i}\right) \subseteq \bigcap_{i \in I} X_{i} \rightarrow \bigcap_{i \in I} Y_{i}
$$

\footnotetext{
${ }^{6}$ It should be noted that I include the combinator names because they are commonly used in the literature on relevant and substructural logics, but that I don't mean by their inclusion to suggest anything deeper about the logics (for instance, I don't mean to claim that they include $\circ$ in the language, or can otherwise represent the central application operation of combinatory logic).

${ }^{7}$ Whenever I write generally about logics, I'll always mean extensions of $\mathbf{F}$.
}

Australasian Journal of Logic (18:1) 2020, Article no. 1 
Proof. Note that $\bigcap_{i \in I}\left(X_{i} \rightarrow Y_{i}\right) \subseteq X_{j} \rightarrow Y_{j}$ for each $j \in I$, and furthermore $X_{j} \rightarrow Y_{j} \subseteq$ $\bigcap_{i \in I} X_{i} \rightarrow Y_{j}$ follows from $(\wedge \mathrm{E})$ and $\left(\mathrm{rB}^{\prime}\right)$ when $j \in I$, so $\bigcap_{i \in I}\left(X_{i} \rightarrow Y_{i}\right) \subseteq \bigcap_{i \in I} X_{i} \rightarrow Y_{j}$ for each $Y_{j}$. It follows that $\bigcap_{i \in I}\left(X_{i} \rightarrow Y_{i}\right) \subseteq \bigcap_{j \in I}\left(\bigcap_{i \in I} X_{i} \rightarrow Y_{j}\right)$, and so $\bigcap_{i \in I}\left(X_{i} \rightarrow Y_{i}\right) \subseteq$ $\bigcap_{i \in I} X_{i} \rightarrow \bigcap_{i \in I} Y_{i}$ follows by $(\wedge \mathrm{I})$.

As a final note, while the first four axioms/rules are valid in all TR frames, the others are not. To state their TR frame correspondents, it is easiest to employ the following standard notational conventions:

$$
\begin{gathered}
R \alpha \beta \gamma \delta:=\exists \epsilon(R \alpha \beta \epsilon \& R \epsilon \gamma \delta) \\
R \alpha(\beta \gamma) \delta:=\exists \epsilon(R \beta \gamma \epsilon \& R \alpha \epsilon \delta)
\end{gathered}
$$

Then the corresponding TR frame constraints are:

- (B)

- $\left(\mathrm{B}^{\prime}\right)$

$$
R \alpha \beta \gamma \delta \Rightarrow R \alpha(\beta \gamma) \delta
$$$$
R \alpha \beta \gamma \delta \Rightarrow R \beta(\alpha \gamma) \delta
$$

For further details on frame correspondence in TR semantics, see [35, 30], and for NTR see $[32,33,17]$.

2.5. Canonical NTR Models. Below I'll be working with the prime theories of various logics, squeezed into an NTR structure - i.e. I'll be working with the canonical models of these logics. This section collects some important facts about these structures.

Definition 2.1. $\alpha$ is an L-theory iff

- $\vdash_{\mathbf{L}} A \rightarrow B$ and $A \in \alpha$ imply $B \in \alpha$

- $A, B \in \alpha$ implies $A \wedge B \in \alpha$

$\alpha$ is prime iff $A \vee B \in \alpha$ only if one of $A, B$ is in $\alpha$.

$\alpha$ is normal iff $\vdash_{\mathbf{L}} A$ implies $A \in \alpha$.

Given a set of formulas $\Gamma$ and a logic $\mathbf{L}$, let $\Gamma$ 's theory-extension be:

$$
[\Gamma)=\left\{B \mid \exists A_{1}, \ldots, A_{n} \in \Gamma\left(\vdash_{\mathbf{L}} \bigwedge_{1 \leq i \leq n} A_{i} \rightarrow B\right)\right\}
$$

Note that $[\Gamma)$ is always at least an L-theory, but there is no guarantee of primeness or normality. The NTR canonical model of a logic $\mathbf{L}$ is a tuple

where:

$$
M_{\mathrm{L}}=\left\langle W_{\mathrm{L}}, N_{\mathrm{L}}, R_{\mathrm{L}}, \operatorname{Prop}_{\mathrm{L}}, V_{\mathrm{L}}\right\rangle
$$

- $W_{\mathbf{L}}$ is the set of theories of $\mathbf{L}$ - when the language of $\mathbf{L}$ includes disjunction, it is the set of all prime theories of $\mathbf{L}^{8}$

- $N_{\mathbf{L}} \subseteq W_{\mathbf{L}}$ is the set of normal elements of $W_{\mathbf{L}}$.

Definition 2.2. Given an $M_{\mathbf{L}}, \llbracket A \rrbracket_{\mathbf{L}}:=\left\{\alpha \in W_{\mathbf{L}} \mid A \in \alpha\right\}$.

${ }^{8}$ Note that in the terminology of $[32,33]$ it is only logics with normal disjunction that are considered here.

Australasian Journal of Logic (18:1) 2020, Article no. 1 
- $R_{\mathrm{L}} \alpha X Y \Longleftrightarrow \exists B, C\left(X=\llbracket B \rrbracket_{\mathrm{L}} \& Y=\llbracket C \rrbracket_{\mathrm{L}} \& B \rightarrow C \in \alpha\right)$

- $\operatorname{Prop}_{\mathbf{L}}=\left\{X \subseteq W_{\mathbf{L}} \mid \exists A\left(X=\llbracket A \rrbracket_{\mathbf{L}}\right)\right\}$

- $V_{\mathbf{L}}(p)=\llbracket p \rrbracket_{\mathbf{L}}$

Proposition 2.4. The following facts are true of canonical NTR models - the proofs are either available in [32] or are immediate:

- $\forall A \exists X \in \operatorname{Prop}_{\mathbf{L}}\left(X=\llbracket A \rrbracket_{\mathbf{L}}\right)$

- $\forall X \in \operatorname{Prop}_{\mathbf{L}} \exists A\left(X=\llbracket A \rrbracket_{\mathbf{L}}\right)$

- $\vdash_{\mathbf{L}} A \rightarrow B \Longleftrightarrow \llbracket A \rrbracket_{\mathbf{L}} \subseteq \llbracket B \rrbracket_{\mathbf{L}}$

The next fact is important for later:

Proposition 2.5. The following are equivalent, for any formulas $A, B$ and any (prime) theories $\alpha, \beta$ :

- $\exists C \in \mathcal{L}(A \rightarrow C \in \alpha \& C \rightarrow B \in \beta)$

- $\exists X \in \operatorname{Prop}_{\mathbf{L}}\left(R_{\mathbf{L}} \alpha \llbracket A \rrbracket_{\mathbf{L}} X \& R_{\mathbf{L}} \beta X \llbracket B \rrbracket_{\mathbf{L}}\right)$

As a shorthand, I'll write the latter as $R_{\mathbf{L}} \beta\left(\alpha \llbracket A \rrbracket_{\mathbf{L}}\right) \llbracket B \rrbracket_{\mathbf{L}}$, following the pattern of $R \alpha(\beta \gamma) \delta$ in the TR semantics.

Finally, the key result is the following.

Proposition 2.6 (Adequacy). If $\mathbf{L}$ is any logic extending $\mathbf{F}$ by any of the axioms/rules listed above, then:

$$
\vDash_{M_{\mathbf{L}}} A \text { iff } \vdash_{\mathbf{L}} A .
$$

A more general result is proved in [32] (they consider more frame correspondents, as well as logics with negation).

\section{Channel COMPOSITION}

Barwise and co-auathors, in a couple of papers in the early 90s [4, 6], developed channel theory in terms of a ternary relation semantics which is, more or less, an extension of TR semantics. So the central ingredient is the relation $R \alpha \beta \gamma$ representing the state of affairs that $\alpha$ is a channel from $\beta$ to $\gamma$, though he represents this ternary relation using (some variation on) the notation $\beta \stackrel{\alpha}{\rightarrow} \gamma$. In particular, he stipulates the existence of two kinds of composite channels, though I'll only be discussing serial composition. The serial composite of channels $\alpha, \beta$ is a channel $\alpha ; \beta$ such that, for any situations $\gamma, \delta$ :

$$
R(\alpha ; \beta) \gamma \delta \Longleftrightarrow R \beta(\alpha \gamma) \delta
$$

This is meant to represent/simulate the effect of linking two channels up in series, and allowing information to flow along the first, and then the second, from the input situation to the output. Given the shorthand above, this does seem to be capturing something like the right idea - there is some situation $\epsilon$ such that $\alpha$ is a channel from $\gamma$ to $\epsilon$, and furthermore $\beta$ is a channel from $\epsilon$ to $\delta$.

In light of this development, both Barwise (and his co-authors Gabbay and Hartonas) [6] and Restall [29] (in the same volume) note that the work of serial composition is easily modeled by the twisted associativity principle $\left(B^{\prime}\right)$ when in this Australasian Journal of Logic (18:1) 2020, Article no. 1 
framework, we identify the serial composition of $\alpha$ with $\beta$ with the result of applying binary 'operation' encoded by the ternary relation to them. ${ }^{9}$ This cannot behave simply functionally, if situations are to be modeled by prime theories, for well known reasons having to do with combining relevant conditionals with distributive lattice operations. So instead we have to understand $R \alpha \beta \gamma$ as the image of the operation which, at the level of propositions, is represented by $A \circ B$. Following Restall [29], I'll write this 'operation' on $\alpha, \beta$ as:

$$
\alpha \circ \beta=\{\gamma \mid R \alpha \beta \gamma\}
$$

and say that $\alpha \circ \beta$ satisfies a formula $A$ when $\alpha \circ \beta \subseteq \llbracket A \rrbracket$ (and the appropriate analogue for any $\left.X \subseteq \wp(W)^{\uparrow}\right)$.

Noteworthy about $\alpha \circ \beta$ is that, given the truth condition for $\rightarrow$, we more or less immediately have:

$$
\alpha \in X \rightarrow Y, \beta \in X \Rightarrow \alpha \circ \beta \subseteq Y
$$

Suggesting that $\circ$ is a natural candidate for modeling the result of applying the conditional information supported by the channel $\alpha$ to that information supported by $\beta{ }^{10}$ A central insight of Barwise, Gabbay, Hartonas, and Restall, is that under the various associativity assumptions (which are desirable for some other reasons), it is a sensible strategy to identify $\alpha ; \beta$ with $\alpha \circ \beta$. It can be shown:

Proposition 3.1. If $\mathbf{L}$ is complete w.r.t. some class of $T R$ frames satisfying $\left(B^{\prime}\right)$, then for any model $M$ and any $X, Y \in \wp\left(W^{M}\right)^{\uparrow}$ :

$$
\alpha \in X \rightarrow Z, \beta \in Z \rightarrow Y \Rightarrow \alpha \circ \beta \subseteq X \rightarrow Y
$$

Proof. Suppose that $R \alpha \beta \gamma$, and furthermore that $R \gamma \delta \epsilon$ and $\delta \in X$. Thus $R \alpha \beta \delta \epsilon$, so by $\left(\mathrm{B}^{\prime}\right), R \beta(\alpha \delta) \epsilon$, and thus there is a $\zeta$ such that $R \alpha \delta \zeta$ and $R \beta \zeta \epsilon$. The former implies that $\zeta \in Z$ and the latter thus implies that $\epsilon \in Y$. So $\gamma \in X \rightarrow Y$.

Of course, this point can be seen just by working with Barwise's original definition, assuming that ; is functional. With that definition $\mathrm{B}^{\prime}$ and its converse $d u a l$ combinator $\mathrm{b}^{\prime}$ provide the desired equivalence:

$$
R \alpha \beta \gamma \delta \Longleftrightarrow R \beta(\alpha \gamma) \delta
$$

The imposition of these structural rules also provides Barwise et al the means to build their target information network structures out of algebraic models of the associative Lambek calculus.

\footnotetext{
${ }^{9}$ The reason why it is $B^{\prime}$ and not $B$ here is that I employ the convention that in the truth condition for $\rightarrow$ using the ternary $R$, the situation at which the conditional formula is evaluated appears in the first argument position. If one instead uses the middle argument position (reserving the first position for that at which the antecedent of the conditional is evaluated), then the transitivity principle at work here is B. There is no substantial difference between these options, and I use the convention I do as it is more standard in the relevant logic literature.

${ }^{10}$ A natural upshot of the failure of the semilattice semantics of [40] is that in the usual TR setting, these sorts of actions on prime theories do not, generally, produce more prime theories.

Australasian Journal of Logic (18:1) 2020, Article no. 1
} 
3.1. Some Reasons to Dislike (B) and $\left(B^{\prime}\right)$. As we've seen, in Restall and Barwise's work the axioms $(B)$ and $\left(B^{\prime}\right)$ play a substantial role. The corresponding frame constraints allow channel composition to be modeled along the same lines as application of a channel to a site. I want to survey some reasons to think that, on the channel-theoretic story, these axioms, and their corresponding frame constraints, are theoretically undesirable.

These axioms, and their corresponding frame constraints, have been criticised in some recent work by Sebastian Sequoiah-Grayson providing epistemic interpretations of the TR semantic framework and closely related structures [37, 38]. His reasons may also, to an extent, be extended to provide reasons to reject them in this setting. Indeed, the idea, pursued below, of considering actions on channels/situations is influenced by Sequoiah-Grayson's notion of an epistemic action. For the most part, he considers abstract counterexamples, but I also think we might be able to get some traction with more concrete counterexamples.

To aid in this, I'll use some diagrams. I'll represent $R \alpha \beta \gamma$, following Barwise $[4,6]$, as an indexed arrow $\beta \stackrel{\alpha}{\longrightarrow} \gamma$, representing the fact that $\alpha$ is a channel between situations $\beta, \gamma$. Using this, we can represent the defined combination $R \alpha \beta \gamma \delta$ with the following diagram - using '.' to pick out the existentially quantified position (in this case, the arrow (channel) itself):

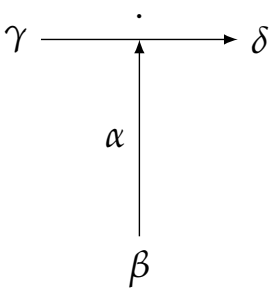

and we can represent $R \alpha(\beta \gamma) \delta$ as:

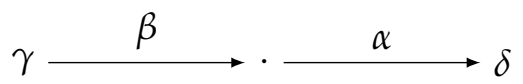

This makes it clear how this latter kind composition of ternary relations models composition of arrows. So the frame constraint for (B) allows one to infer the latter from the former, and that for $\left(\mathrm{B}^{\prime}\right)$ allows us to infer from the former the variation on the latter where the positions of $\alpha, \beta$ are permuted. For the second, note that we must treat $\beta$ as behaving like a site in the first diagram, but as a channel in the second. It has come under some discussion $[6,29]$ whether we should understand channels as separate kinds of thing from sites. Treating them as separate would seem to me to provide good reason to rule out $\left(B^{\prime}\right)$ immediately.

However, even if we take channels and sites just to all be situations, it is still substantial to posit these systematic interactions between situations. On a plausible way of understanding versions of the account where channels and sites are of the same kind, we should talk of situations acting as a channel in some circumstance (as, for instance, when we discuss applying conditional information holding in that Australasian Journal of Logic (18:1) 2020, Article no. 1 
situation to some other situation) or talk of them acting as sites - the context will determine what situation plays what role where. Positing $\left(\mathrm{B}^{\prime}\right)$ amounts to demanding that whenever $\alpha$, acting as a channel, applied to $\beta$ produces some situation which acts as a channel from $\gamma$ to $\delta$, it must also be that $\beta$ and $\alpha$, acting as channels, can be serially composed to get from $\gamma$ to $\delta$. Perhaps there's some argument to think that situations must behave this way, but it strikes me as very far from obvious.

I take it that the proponent of $\left(\mathrm{B}^{\prime}\right)$ has the onus to justify the inclusion of their desired principle, but it would still be nice to have a compelling counterexample. To that end, consider the following:

Counterexample Consider a cable $\alpha$ taking some instructions $\beta$ to a motor which, upon receiving them, will open a window, thus forming a channel allowing for information from the situation immediately outside the window $\gamma$ to flow into the room $\delta$. This is a connection of of the form $R \alpha \beta \gamma \delta$.

Question: if we take the instructions $\beta$ and compose them with the cable $\alpha$, do we get a channel taking $\gamma$ to $\delta$ ? In other words, if we apply (the conditional information) in $\beta$ to any appropriate information in $\gamma$, and take the resulting situation and apply the conditional information in $\alpha$ to it, do we get just $\delta$ ? That is, does it follow that $R \alpha(\beta \gamma) \delta$ ?

One consideration in favour of ' $\mathrm{No}^{\prime}$ is that it's not obvious what it would mean to compose some instructions and a cable which carries those instructions. One can consider the result of applying $\alpha$ to $\beta$ by sending $\beta$ to the resulting channel, but this doesn't seem to involve conditional information supported by $\beta$ at all, as composing seems to call for. The connection $R \alpha \beta \gamma \delta$ makes perfect sense, but, on the face of it, the connection $R \beta(\alpha \gamma) \delta$ doesn't make sense, without filling in more information about the information supported by $\beta$. The important point is just that the latter doesn't follow from the former.

3.2. Another Way to Proceed. The method in [39] suggests another way to characterise channel composition - in terms of the conditional information supported by channels, rather than by relations among channels themselves.

Definition 3.1. L admits channel composition just in case for any pair $\alpha, \beta$ of (prime) L-theories, there is another, $\gamma$, such that for any formulas $A, B$ :

$$
A \rightarrow B \in \gamma \Longleftrightarrow \exists C(A \rightarrow C \in \alpha \& C \rightarrow B \in \beta)
$$

Note that if $\mathbf{L}$ admits channel composition, then while not all of its models must include composite points, it is complete with respect to the set of its models which do.

Note that this definition is stronger than that initially proposed in [4], where only the right-to-left condition is required. However, it seems to me natural to make this Australasian Journal of Logic (18:1) 2020, Article no. 1 
more stringent demand, as if such $\gamma^{\prime}$ s can be shown to exist, then we'll have pinned down something closer to the channel composites of the various $\alpha, \beta .^{11}$

\section{Some Sufficient Conditions For Channel Composition}

In this section, I'll display two sufficient conditions for a logic $\mathbf{L}$ to admit channel composition in the sense of $\S 3.2^{12}$

4.1. Bubbling. The first is that the logic satisfy the Bubbling Lemma, initially proved [2] for $\mathbf{B}^{\wedge \top}$, the extension of $\mathbf{B}^{\wedge}$ by the addition of the propositional constant $T$, as discussed in [30]. This lemma is property-ified as follows:

Definition 4.1. A logic $\mathbf{L}$ (whose language extends $\{\rightarrow, \wedge\}$ ) bubbles iff whenever

$$
\vdash_{\mathbf{L}} \bigwedge_{i \in I}\left(A_{i} \rightarrow B_{i}\right) \rightarrow(C \rightarrow D)
$$

(and $\nvdash_{\mathbf{L}} \top \rightarrow D$, if the language of $\mathbf{L}$ contains $\top$ ) then there is a non-empty $J \subseteq I$ such that:

$$
\vdash_{\mathbf{L}} C \rightarrow \bigwedge_{j \in J} A_{j} \quad \text { and } \quad \vdash_{\mathbf{L}} \bigwedge_{j \in J} B_{j} \rightarrow D .
$$

Briefly, when L bubbles, one can decompose certain validities containing nested conditionals into other validities containing at least one fewer such nesting. This is a very nice property which provides the grist for natural theory constructions. The canonical application of the bubbling lemma for $\mathbf{B}^{\wedge \top}$ is in providing nice filter models of the $\lambda$-calculus (with intersection types) and combinatory logic. In the relevant logic literature, Meyer and a number of co-authors over the years (see $[16,14,28])$ used this fact to build nice ternary relation models of combinatory logic. ${ }^{13}$

\subsection{ECP.}

Definition 4.2. A logic $\mathbf{L}$ in a language extending $\{\rightarrow, \wedge, \vee\}$ has the entailmentconsequent-primeness property (or ECP) just in case whenever:

$$
\vdash_{\mathbf{L}} \bigwedge_{i \in I}\left(A_{i} \rightarrow B_{i}\right) \rightarrow \bigvee_{j \in J}\left(C_{j} \rightarrow D_{j}\right)
$$

then there is a $j \in J$ s.t.

$$
\vdash_{\mathbf{L}} \bigwedge_{i \in I}\left(A_{i} \rightarrow B_{i}\right) \rightarrow\left(C_{j} \rightarrow D_{j}\right)
$$

This property is a special instance of a more natural property delivered by the combination of the disjunction property:

\footnotetext{
${ }^{11}$ Restall [29] obtains a similar 'as unique as possible' construction for channel composites (that is, unique with respect to the theory, though not necessarily with whatever prime theories extend that theory) in the context with associativity.

${ }^{12}$ For my purposes the logics of interest from here on all extend $\mathbf{B}^{\wedge}$, so I take $\mathbf{L}$ to range over just those axiomatic extensions.

${ }^{13}$ Note that in $[3, \mathrm{p} .624]$, bubbling logics are called $\beta$-sound.
}

Australasian Journal of Logic (18:1) 2020, Article no. 1 


$$
\text { Whenever } \vdash_{\mathbf{L}} A \vee B \text {, either } \vdash_{\mathbf{L}} A \text { or } \vdash_{\mathbf{L}} B
$$

and the admissibility of a rule familiar from Harrop [18] (where $A$ is required to contain no relevant instance of disjunction):

$$
A \rightarrow B \vee C \Rightarrow(A \rightarrow B) \vee(A \rightarrow C)
$$

\subsection{Putting the Sufficient Conditions to Work.}

Theorem 4.1. Let $\mathbf{L}$ be a logic extending $\mathbf{B}^{\wedge}$ with (possibly) a larger set of connectives. Then if $\mathbf{L}$ bubbles, then for any pair of $\mathbf{L}$-theories $\alpha, \beta$ (prime if the language of $\mathbf{L}$ extends $\left.\mathcal{L}_{[\mathrm{V}]}\right)$, there is a theory $\alpha ; \beta$ such that:

$$
A \rightarrow B \in \alpha ; \beta \Longleftrightarrow R_{\mathbf{L}} \beta\left(\alpha \llbracket A \rrbracket_{\mathbf{L}}\right) \llbracket B \rrbracket_{\mathbf{L}}
$$

Proof. Define $(\alpha ; \beta)_{0}:=\left\{A \rightarrow B \mid R_{\mathbf{L}} \beta\left(\alpha \llbracket A \rrbracket_{\mathbf{L}}\right) \llbracket B \rrbracket_{\mathbf{L}}\right\}$, and let $\alpha ; \beta=\left[(\alpha ; \beta)_{0}\right)$. It is immediate that $\alpha ; \beta$ is a theory, and furthermore if $R_{\mathbf{L}} \beta\left(\alpha \llbracket A \rrbracket_{\mathbf{L}}\right) \llbracket B \rrbracket_{\mathbf{L}}$, then $A \rightarrow B \in$ $\alpha ; \beta$, so I prove the converse.

Suppose that $A \rightarrow B \in \alpha ; \beta$. Then there are formulas $C_{1}^{\prime}, \ldots, C_{n}^{\prime} \in(\alpha ; \beta)_{0}$ such that $\vdash_{\mathbf{L}} \bigwedge_{i \in I} C_{i}^{\prime} \rightarrow(A \rightarrow B)$ where $I=\{1, \ldots, n\}$. By definition, each $C_{i}^{\prime}$ is of the form $C_{i} \rightarrow D_{i}$ where $R_{\mathbf{L}} \beta\left(\alpha \llbracket C_{i} \rrbracket_{\mathbf{L}}\right) \llbracket D_{i} \rrbracket_{\mathbf{L}}$. Thus, since $\mathbf{L}$ bubbles, there is a non-empty $J \subseteq I$ such that:

$$
\vdash_{\mathbf{L}} A \rightarrow \bigwedge_{j \in J} C_{j} \quad \text { and } \quad \vdash_{\mathbf{L}} \bigwedge_{j \in J} D_{j} \rightarrow B
$$

It follows that $\llbracket A \rrbracket_{\mathbf{L}} \subseteq \llbracket \bigwedge_{j \in J} C_{j} \rrbracket_{\mathbf{L}}=\bigcap_{j \in J} \llbracket C_{j} \rrbracket_{\mathbf{L}}$ and also that $\bigcap_{j \in J} \llbracket D_{j} \rrbracket_{\mathbf{L}}=\llbracket \bigwedge_{j \in J} D_{j} \rrbracket_{\mathbf{L}} \subseteq \llbracket B \rrbracket_{\mathbf{L}}$. Furthermore, for every $C_{j} \rightarrow D_{j}$ there is an $E_{j}$ such that $C_{j} \rightarrow E_{j} \in \alpha$ and $E_{j} \rightarrow$ $D_{j} \in \beta$. By Prop 2.3, and the adequacy theorem, $\alpha \in \bigcap_{j \in J} \llbracket C_{j} \rrbracket_{\mathbf{L}} \rightarrow \bigcap_{j \in J} \llbracket E_{j} \rrbracket_{\mathbf{L}}$ and $\beta \in \bigcap_{j \in J} \llbracket E_{j} \rrbracket_{\mathbf{L}} \rightarrow \bigcap_{j \in J} \llbracket D_{j} \rrbracket_{\mathbf{L}}$. Let $X=\bigcap_{j \in J} \llbracket E_{j} \rrbracket_{\mathbf{L}}$. Since $\llbracket A \rrbracket_{\mathbf{L}} \subseteq \bigcap_{j \in J} \llbracket C_{j} \rrbracket_{\mathbf{L}}$, by $\left(\mathrm{rB}^{\prime}\right)$ :

$$
\bigcap_{j \in J} \llbracket C_{j} \rrbracket_{\mathbf{L}} \rightarrow X \subseteq \llbracket A \rrbracket_{\mathbf{L}} \rightarrow X
$$

Similarly, since $\bigcap_{j \in J} \llbracket D_{j} \rrbracket_{\mathbf{L}} \subseteq \llbracket B \rrbracket_{\mathbf{L}}$, by $(\mathrm{rB})$ :

$$
X \rightarrow \bigcap_{j \in J} \llbracket D_{j} \rrbracket_{\mathbf{L}} \subseteq X \rightarrow \llbracket B \rrbracket_{\mathbf{L}}
$$

Thus, $\alpha \in \llbracket A \rrbracket_{\mathbf{L}} \rightarrow X$ and $\beta \in X \rightarrow \llbracket B \rrbracket_{\mathbf{L}}$, and thus $R_{\mathbf{L}} \beta\left(\alpha \llbracket A \rrbracket_{\mathbf{L}}\right) \llbracket_{B} \rrbracket_{\mathbf{L}}$.

Theorem 4.2. Let $\mathbf{L}$ be a logic in a language expanding $\mathcal{L}_{[\mathrm{V}]}$, and furthermore suppose that $\mathbf{L}$ bubbles and has ECP. Then there is a prime theory $(\alpha ; \beta)^{\prime}$ such that:

$$
A \rightarrow B \in(\alpha ; \beta)^{\prime} \Longleftrightarrow R_{\mathbf{L}} \beta\left(\alpha \llbracket A \rrbracket_{\mathbf{L}}\right) \llbracket B \rrbracket_{\mathbf{L}}
$$

Proof. We can use the construction of the previous theorem to build a theory $\alpha ; \beta$ satisfying the target property. Now to obtain a prime $(\alpha ; \beta)^{\prime} \supseteq \alpha ; \beta$, we can employ Belnap's Pair Extension lemma (details are available in [30]). For note that the following is an independent pair of sets of formulas: Australasian Journal of Logic (18:1) 2020, Article no. 1 


$$
\left\langle\alpha ; \beta,\left\{C \rightarrow D \mid \overline{R_{\mathbf{L}}} \beta\left(\alpha \llbracket C \rrbracket_{\mathbf{L}}\right) \llbracket D \rrbracket_{\mathbf{L}}\right\}\right\rangle
$$

For suppose that there were $A_{1}^{\prime}, \ldots, A_{m^{\prime}}^{\prime} \in \alpha ; \beta$ and $C_{1} \rightarrow D_{1}, \ldots, C_{n} \rightarrow D_{n}$ such that $\vdash_{\mathbf{L}} \bigwedge_{1 \leq i \leq m^{\prime}}^{\wedge} A_{i}^{\prime} \rightarrow \bigvee_{1 \leq j \leq n}^{\bigvee}\left(C_{j} \rightarrow D_{j}\right)$. By construction, each $A_{i}^{\prime}$ is entailed by a finite conjunction of conditionals, so using $(\mathrm{rB}),\left(\mathrm{rB}^{\prime}\right)$, it follows that:

$$
\vdash_{\mathbf{L}} \bigwedge_{1 \leq i \leq m}\left(A_{i} \rightarrow B_{i}\right) \rightarrow \bigvee_{1 \leq j \leq n}\left(C_{j} \rightarrow D_{j}\right)
$$

By ECP, there is a $j \in J$ such that $\vdash_{\mathbf{L}} \bigwedge_{1 \leq i \leq m}\left(A_{i} \rightarrow B_{i}\right) \rightarrow\left(C_{j} \rightarrow D_{j}\right)$, and thus $C_{j} \rightarrow D_{j} \in \alpha ; \beta$ and so, by Theorem $4.1, R_{\mathbf{L}} \beta\left(\alpha \llbracket C_{j} \rrbracket_{\mathbf{L}}\right) \llbracket D_{j} \rrbracket_{\mathrm{L}}$, contrary to the assumption. Therefore, the above pair is a pair, and so by the pair extension lemma, can be extended to a full pair with the left element $(\alpha ; \beta)^{\prime}$ a prime theory extending $\alpha ; \beta$ but containing no $C \rightarrow D$ such that $\overline{R_{\mathbf{L}}} \beta\left(\alpha \llbracket C \rrbracket_{\mathbf{L}}\right) \llbracket D \rrbracket_{\mathbf{L}}$. So $(\alpha ; \beta)^{\prime}$ is the desired prime theory.

Theorem 4.3. The following hold of the construction of $\alpha ; \beta$ from Thm 4.1:

(1) If $\alpha, \beta \in N$ then $\alpha ; \beta \in N$

(2) If $A \rightarrow B \in \alpha$ and $\beta \in N$ then $A \rightarrow B \in \alpha ; \beta$

(2a) If $\alpha \in N$ and $A \rightarrow B \in \beta$ then $A \rightarrow B \in \alpha ; \beta$

(3) $A \rightarrow B \in \alpha ;(\beta ; \gamma)$ iff $A \rightarrow B \in(\alpha ; \beta) ; \gamma$

Proof. (1) For this, note that if $\alpha, \beta \in N$, then if $\vdash A \rightarrow B$ then $A \rightarrow B \in \alpha$ and $B \rightarrow B \in \beta$, and thus $A \rightarrow B \in \alpha ; \beta$.

(2), (2a) are left to the interested reader to verify.

(3) For this, we show that $\left(\alpha ;(\beta ; \gamma)_{0}\right)_{0}=\left((\alpha ; \beta)_{0} ; \gamma\right)_{0}$. This follows from the following sequence of equivalences - recall that we have shown $A \rightarrow B \in \alpha ; \beta \Longleftrightarrow$ $A \rightarrow B \in(\alpha ; \beta)_{0}$.

$$
\begin{aligned}
A \rightarrow B \in \alpha ;(\beta ; \gamma) & \Longleftrightarrow A \rightarrow B \in(\alpha ;(\beta ; \gamma))_{0} \\
& \Longleftrightarrow \exists C(A \rightarrow C \in \alpha \& C \rightarrow B \in \beta ; \gamma) \\
& \Longleftrightarrow \exists C\left(A \rightarrow C \in \alpha \& C \rightarrow B \in(\beta ; \gamma)_{0}\right) \\
& \Longleftrightarrow \exists C, D(A \rightarrow C \in \alpha \& C \rightarrow D \in \beta \& D \rightarrow B \in \gamma) \\
& \Longleftrightarrow \exists D\left(A \rightarrow D \in(\alpha ; \beta)_{0} \& D \rightarrow B \in \gamma\right) \\
& \Longleftrightarrow \exists D(A \rightarrow D \in \alpha ; \beta \& D \rightarrow B \in \gamma) \\
& \Longleftrightarrow A \rightarrow B \in((\alpha ; \beta) ; \gamma)_{0} \\
& \Longleftrightarrow A \rightarrow B \in(\alpha ; \beta) ; \gamma
\end{aligned}
$$

In short, while it may not be that $\alpha ; \beta ; \gamma)$ and $(\alpha ; \beta) ; \gamma$ agree on every formula, they do agree everywhere we care about - in the implication formulas.

\section{CAndidate Channel-Admitting Logics}

This leaves the question of which logics satisfy these sufficient conditions. Sadly there are almost none that do, but I'll present some evidence (though not a proof) that there really are almost none. For indeed, there is one promising candidate!

Australasian Journal of Logic (18:1) 2020, Article no. 1 
5.1. $\mathbf{B B}_{(\wedge \mathbf{I})}^{+}$. Recall that $\mathbf{B B}_{(\wedge \mathrm{I})}^{+}$extends $\mathbf{B B}^{+}$by the axiom $(\wedge \mathrm{I})$, and so extends the basic logic $\mathbf{F}^{+}$by this axiom, as well as by $(\mathrm{rB})$ and $\left(\mathrm{rB}^{\prime}\right)$.

Theorem 5.1. $\mathbf{B B}_{(\wedge I)}^{+}$has ECP.

Proof. By an application of the method of metavaluations [24, 13], it can be shown that $\mathbf{B B}_{(\wedge \mathrm{I})}^{+}$is metacomplete, and thus has the disjunction property. ${ }^{14}$ It suffices, then, to show that the following rule is admissible in $\mathbf{B B}_{(\wedge \mathrm{I})}^{+}$:

$$
\bigwedge_{i \in I}\left(A_{i} \rightarrow B_{i}\right) \rightarrow \bigvee_{j \in J}\left(C_{j} \rightarrow D_{j}\right) \Rightarrow \bigvee_{j \in J}\left(\bigwedge_{i \in I}\left(A_{i} \rightarrow B_{i}\right) \rightarrow\left(C_{j} \rightarrow D_{j}\right)\right)
$$

To that end, let $E=\bigwedge_{i \in I}\left(A_{i} \rightarrow B_{i}\right)$, and suppose that there is a $\mathbf{B B}_{(\wedge \mathrm{I})}^{+}$model $M$ such that $\not \nvdash_{M} \underset{j \in J}{\bigvee}\left(E \rightarrow\left(C_{j} \rightarrow D_{j}\right)\right)$. So then where $M=\langle W, N, R$, Prop,$V\rangle$, we have some $\alpha_{1}, \ldots, \alpha_{n} \in \llbracket E \rrbracket_{M}(J=\{1, \ldots, n\})$ where $\alpha_{j} \notin \llbracket C_{j} \rightarrow D_{j} \rrbracket_{M}$ holds for each $j \in J$. The aim is to build a model $M^{\prime}$ with a point $\alpha$ such that $\alpha \in \llbracket E \rrbracket_{M^{\prime}}$ and $\alpha \notin \bigcup_{j \in J} \llbracket C_{j} \rightarrow D_{j} \rrbracket_{M^{\prime}}$, hence showing that $E \rightarrow \bigvee_{j \in J}\left(C_{j} \rightarrow D_{j}\right)$ is not a $\mathbf{B B}_{(\wedge \mathrm{I})}^{+}$-theorem.

So, let's build $M^{\prime}$ out of $M$ as follows:

- $W^{\prime}=W \cup\{\alpha\}$

- $N^{\prime}=N$

The other elements of $M^{\prime}$ will have to be defined inductively. Let $V^{\prime}$ be the least function of type $\mathbb{P} \longrightarrow \wp\left(W^{\prime}\right)$ such that:

(a) For $\beta \in W, \beta \in V^{\prime}(p)$ if $\beta \in V(p)$

(b) $\alpha \in V^{\prime}(p)$ if $\forall j \in J\left(\alpha_{j} \in V(p)\right)$

Now the trick is to build $R^{\prime}$ and Prop $p^{\prime}$ to ensure that these satisfy the model constraints, and have a couple extra properties. These we construct by induction.

Fix $\operatorname{Prop}_{0}^{\prime}=\left\{X \subseteq W^{\prime} \mid X=V^{\prime}(p)\right\}$.

Fix $\langle\beta, X, Y\rangle \in R_{0}^{\prime}$ if $X, Y \in \operatorname{Prop}_{0}^{\prime}$ (so fix $X=\llbracket p \rrbracket_{M^{\prime}}$ and $Y=\llbracket q \rrbracket_{M^{\prime}}$ ) and either:

(c) $\beta \in W$ and $R \beta \llbracket p \rrbracket_{M \llbracket q \rrbracket_{M}}$

(d) $\beta=\alpha$ and $\forall j \in J\left(R \alpha_{j} \llbracket p \rrbracket_{M} \llbracket q \rrbracket_{M}\right)$

Then, fix:

$\operatorname{Prop}_{n+1}^{\prime}=\operatorname{Prop}_{n}^{\prime} \cup\left\{X \subseteq W^{\prime} \mid \exists Y, Z \in \operatorname{Prop}_{n}^{\prime}(X=Y \cup Z, Y \cap Z\right.$, or $\left.Y \rightarrow Z)\right\}$

and further, let $\langle\beta, X, Y\rangle \in R_{n+1}^{\prime}$ if $X, Y \in \operatorname{Prop}_{n}^{\prime}$ (so there are $A, B \in \mathcal{L}$ such that $\left.X=\llbracket A \rrbracket_{M^{\prime}}, Y=\llbracket B \rrbracket_{M^{\prime}}\right)$ and either:

(c') $\beta \in W$ and $R \beta \llbracket A \rrbracket_{M} \llbracket B \rrbracket_{M}$

(d') $\beta=\alpha$ and $\forall j \in J\left(R \alpha_{j} \llbracket A \rrbracket_{M} \llbracket B \rrbracket_{M}\right)$

\footnotetext{
${ }^{14}$ While $\mathbf{B B}_{(\wedge \mathrm{I})}^{+}$is not among the systems directly covered in [24], it is straightforward to adapt the arguments to show that it is metacomplete - the only rule not accounted for there is ( $\mathrm{VE}$ ), but the argument needed for this rule is similar to those given in Meyer's paper.

Australasian Journal of Logic (18:1) 2020, Article no. 1
} 
and set Prop $^{\prime}=\bigcup_{n \in \mathbb{N}} \operatorname{Prop}_{n}^{\prime}, R^{\prime}=\bigcup_{n \in \mathbb{N}} R_{n}^{\prime}$.

Note that it follows immediately that $V^{\prime}: \mathbb{P} \longrightarrow$ Prop $^{\prime}$ and that Prop $p^{\prime}$ is closed w.r.t. $\cup, \cap, \rightarrow$. So (C0) is satisfied. Furthermore, we have the following lemmata more or less immediately.

Lemma 5.2. $\forall X \in \operatorname{Prop}^{\prime} \exists A \in \mathcal{L}\left(X=\llbracket A \rrbracket_{M^{\prime}}\right)$

Lemma 5.3. If $\beta \in W$ then $\beta \in \llbracket A \rrbracket_{M^{\prime}} \Longleftrightarrow \beta \in \llbracket A \rrbracket_{M}$

Proof. From the definition of $V^{\prime}$ it follows that $\beta \in V^{\prime}(p) \Longleftrightarrow \beta \in V(p)$ for all $p \in \mathbb{P}$. Furthermore, note that $\beta \in \llbracket A \rightarrow B \rrbracket_{M^{\prime}} \Longleftrightarrow R^{\prime} \beta \llbracket A \rrbracket_{M^{\prime}} \llbracket B \rrbracket_{M^{\prime}}$, which holds only in case $R_{n}^{\prime} \beta \llbracket A \rrbracket_{M^{\prime}} \llbracket B \rrbracket_{M^{\prime}}$, and thus $R \beta \llbracket A \rrbracket_{M} \llbracket B \rrbracket_{M}$. These are the two cases of an induction which pose any problem - the inductive hypothesis takes care of the rest.

Lemma 5.4. $\alpha \in \llbracket A \rrbracket_{M^{\prime}} \Longleftrightarrow \forall j \in J\left(\alpha_{j} \in \llbracket A \rrbracket_{M}\right)$

Proof. As before, we can prove this by induction. The base case is taken care of by the definition of $V^{\prime}$, and the case where $A$ is a conditional formula is taken care of by the definition of $R^{\prime}$. The other cases are similar.

With these, we turn to the rest of the verification of the model constraints. For $(\mathrm{C} 1)-N^{\prime} \subseteq X \rightarrow Y \Longleftrightarrow X \subseteq Y$ for all $X, Y \in$ Prop $^{\prime}$ - note that if $X, Y \in$ Prop $^{\prime}$ there are $A, B \in \mathcal{L}$ such that $X=\llbracket A \rrbracket_{M^{\prime}}, Y=\llbracket B \rrbracket_{M^{\prime}}$. First, suppose that $\llbracket A \rrbracket_{M^{\prime}} \subseteq$ $\llbracket B \rrbracket_{M^{\prime}}$. In this case, by Lem. 5.2, it follows immediately that $\llbracket A \rrbracket_{M} \subseteq \llbracket B \rrbracket_{M}$, and so $N \subseteq \llbracket A \rightarrow B \rrbracket_{M}$. But if $\beta \in N^{\prime}$ then $\beta \in N$, so then $\beta \in \llbracket A \rightarrow B \rrbracket_{M} \Longleftrightarrow \beta \in \llbracket A \rightarrow$ $B \rrbracket_{M^{\prime}}=\llbracket A \rrbracket_{M^{\prime}} \rightarrow \llbracket B \rrbracket_{M^{\prime}}$, as desired.

Next, suppose that $N^{\prime} \subseteq \llbracket A \rightarrow B \rrbracket_{M^{\prime}}$, and let $\beta \in \llbracket A \rrbracket_{M^{\prime}}$. Again, we have for all $\gamma \in N$, that $\gamma \in N^{\prime}$ and that $\gamma \in \llbracket A \rightarrow B \rrbracket_{M} \Longleftrightarrow \gamma \in \llbracket A \rightarrow B \rrbracket_{M^{\prime}}$. Thus, $N \subseteq \llbracket A \rightarrow B \rrbracket_{M^{\prime}}$ and so $\llbracket A \rrbracket_{M^{\prime}} \subseteq \llbracket B \rrbracket_{M^{\prime}}$. Suppose that $\beta \in W$, then the result is an immediate consequence of Lem. 5.2. Suppose $\beta=\alpha$, then by Lem 5.4, it follows that:

$$
\beta \in \llbracket A \rrbracket_{M^{\prime}} \Longleftrightarrow \forall j \in J\left(\alpha_{j} \in \llbracket A \rrbracket_{M}\right) \Rightarrow \forall j \in J\left(\alpha_{j} \in \llbracket B \rrbracket_{M}\right) \Longleftrightarrow \beta \in \llbracket A \rrbracket_{M^{\prime}}
$$

and the result follows.

What remains are the constraints specific to $\mathbf{B B}_{(\wedge \mathrm{I})}^{+}$. These are $(\mathrm{rB}),\left(\mathrm{rB}^{\prime}\right)$, and $(\wedge \mathrm{I})$. The first two are similar, so I'll only present one of those, and sketch the argument for the third.

For (rB), suppose that $X, Y, Z \in$ Prop $^{\prime}$, so that there are $A, B, C \in \mathcal{L}$ such that $X=\llbracket A \rrbracket_{M^{\prime}}, Y=\llbracket B \rrbracket_{M^{\prime}}$, and $Z=\llbracket C \rrbracket_{M^{\prime}}$. Suppose $\llbracket A \rrbracket_{M^{\prime}} \subseteq \llbracket B \rrbracket_{M^{\prime}}$, and let $\beta \in$ $\llbracket C \rightarrow A \rrbracket_{M^{\prime}}$. If $\beta \in W$, this holds iff $\beta \in \llbracket C \rightarrow A \rrbracket_{M}$, and thus $\beta \in \llbracket C \rightarrow B \rrbracket_{M}$ since $M$ is a $\mathbf{B B}_{(\wedge \mathrm{I})}^{+}$model, which in turn holds iff $\beta \in \llbracket C \rightarrow B \rrbracket_{M^{\prime}}$. If $\beta=\alpha$, then $\beta \in \llbracket C \rightarrow A \rrbracket_{M^{\prime}}$ iff $\forall j \in J\left(\alpha_{j} \in \llbracket C \rightarrow A \rrbracket_{M}\right)$, in which case $\forall j \in J\left(\alpha_{j} \in \llbracket C \rightarrow B \rrbracket_{M}\right)$, and thus $\beta \in \llbracket C \rightarrow B \rrbracket_{M^{\prime}}$, as desired.

Australasian Journal of Logic (18:1) 2020, Article no. 1 
Finally, for $(\wedge \mathrm{I})$, suppose that $X, Y, Z$ are as before, and furthermore that $\beta \in$ $(X \rightarrow Y) \cap(X \rightarrow Z)$. As before, if $\beta \in W$, then $\beta \in X \rightarrow(Y \cap Z)$ can be obtained by Lem 5.2, and if $\beta=\alpha$ the result follows by Lem 5.4.

So $M^{\prime}$ is a $\mathbf{B B}_{(\wedge I)}^{+}$model, and furthermore the construction guarantees that $\alpha \in$ $\llbracket E \rrbracket_{M^{\prime}}$ and $\alpha \notin \bigcup_{j \in J} \llbracket C_{j} \rightarrow D_{j} \rrbracket_{M^{\prime}}$, so $\nvdash_{M^{\prime}} \bigwedge_{i \in I}\left(A_{i} \rightarrow B_{i}\right) \rightarrow \bigvee_{j \in J}\left(C_{j} \rightarrow D_{j}\right)$. So the desired rule is indeed admissible in $\mathbf{B B}_{(\wedge I)}^{+}$.

It follows from this and the fact that $\mathbf{B B}_{(\wedge \mathrm{I})}^{+}$has the disjunction property that $\mathbf{B B}_{(\wedge \mathrm{I})}^{+}$has ECP.

This leaves the last part, which is still open:

Conjecture 5.5. $\mathbf{B B}_{(\wedge I)}^{+}$bubbles.

I don't have a proof to provide here, but I will provide some reasons to think that this conjecture is true.

First, an alternative axiomatisation of $\mathbf{B B}_{(\wedge \mathrm{I})}^{+}$is helpful. The system is a binary assertional system in the terminology of [15] or a FMLA-FMLA system in that of [19]. A sequent here is a pair $\langle A, B\rangle \in \mathcal{L}^{2}$, which I'll write $A \leq B$. I'll occasionally refer to formula in the position of $A$ as the 'precedent' and that in the position of $B$ as the 'succedent.' The following system is adapted from that for ITD/ $\mathbf{B}^{\wedge \top}$ given in $[2,14]$ and elsewhere. It consists of axioms (A1)-(A5) and rules (R1)-(R6):

(A1) $A \leq A$

(A2) $A \wedge B \leq A, \quad A \wedge B \leq B$

(A3) $(A \rightarrow B) \wedge(A \rightarrow C) \leq A \rightarrow B \wedge C$

(A4) $A \leq A \vee B, \quad B \leq A \vee B$

(A5) $A \wedge(B \vee C) \leq(A \wedge B) \vee(A \wedge C)$

(R1) $A \leq C, C \leq B \Rightarrow A \leq B$

(R2) $A \leq A^{\prime}, B \leq B^{\prime} \Rightarrow A \wedge B \leq A^{\prime} \wedge B^{\prime}$

(R3) $A \leq A^{\prime}, B \leq B^{\prime} \Rightarrow A \vee B \leq A^{\prime} \vee B^{\prime}$

(R4) $A^{\prime} \leq A, B \leq B^{\prime} \Rightarrow A \rightarrow B \leq A^{\prime} \rightarrow B^{\prime}$

(R5) $A \leq B, A \leq C \Rightarrow A \leq B \wedge C$

(R6) $A \leq C, B \leq C \Rightarrow A \vee B \leq C$

I'll write $\vdash_{B A} A \leq B$ to indicate that there is a proof of the sequent from the above proof system ("BA" for "binary assertional"). The following result is straightforward.

Proposition 5.6. $\vdash_{B A} A \leq B$ iff $\vdash_{\mathbf{B B}_{(\wedge I)}^{+}} A \rightarrow B$.

This fact, in addition to the following:

- $\vdash_{\mathbf{B B}_{(\wedge \mathrm{I})}^{+}} A \wedge B \Longleftrightarrow \vdash_{\mathbf{B B}_{(\wedge \mathrm{I})}^{+}} A$ and $\vdash_{\mathbf{B B}_{(\wedge \mathrm{I})}^{+}} B$

- $\vdash_{\mathbf{B B}_{(\wedge \mathrm{I})}^{+}} A \vee B \Longleftrightarrow \vdash_{\mathbf{B B}_{(\wedge \mathrm{I})}^{+}} A$ or $\vdash_{\mathbf{B B}_{(\wedge \mathrm{I})}^{+}} B$

Australasian Journal of Logic (18:1) 2020, Article no. 1 
shows that the binary assertional system captures the same theorems as the Hilbert system. This is because any theorem is either a conditional formula (which is covered by the proposition) or is a conjunction or disjunction thereof, which we can simulate, via the above facts, by collections of provable implication formulas. Finally, we can characterise bubbling in this system as the property that whenever:

$$
\bigwedge_{i \in I}\left(A_{i} \rightarrow B_{i}\right) \leq C \rightarrow D
$$

then there is a nonempty $J \subseteq I$ such that:

$$
C \leq \bigwedge_{j \in J} A_{j} \text { and } \bigwedge_{j \in J} B_{j} \leq D
$$

While I won't prove this result (for lack of such a proof), I will provide some evidence which, I suspect, when assembled the right way does provide a proof. On its own, I take the following facts as evidence that $\mathbf{B B}_{(\wedge \mathrm{I})}^{+}$bubbles.

Proposition 5.7. No instance of any axiom of the binary assertional system is a counterexample to bubbling.

Proof. By inspection.

Proposition 5.8. No application of (R2)-(R6) produces a counterexample to bubbling.

Proof. This takes a bit more spelling out, but is mostly by inspection. First note that (R2), (R3), (R5), and (R6) have conclusion sequents which have no instances of the form $\bigwedge_{i \in I}\left(A_{i} \rightarrow B_{i}\right) \leq C \rightarrow D$, so none of these can produce counterexamples. As for (R4), we can obtain something of the right form, but where $I$ is a singleton: $A \rightarrow B \leq C \rightarrow D$, and we can only get there when we've proved $C \leq A$ and $B \leq D$, which works.

So, as usual, (R1) is the troublemaker. It seems that some kind of cut-elimination style argument is called for here, but the details seem to be devilish. What I'll present here are some 'low-in-the-induction' cases, showing that very shorts proof in the binary assertional system don't produce counterexamples to bubbling. I'll consider, first, the application of (R1) to a pair of instances of axioms, showing that no counterexample can arise there, and then go on to consider a case where one of the premises to (R1) is the result of applying (R1) to axioms, and so that also there no counterexamples can arise.

In order to state these results more clearly, I'll need some notation. We are after applications of (R1) which can produce conclusions of the form (Con) $\bigwedge_{i \in I}\left(A_{i} \rightarrow\right.$ $\left.B_{i}\right) \leq C \rightarrow D$, so we are interested in a left premise (Lp), a right premise (Rp), cut together on a cut formula, as below:

$$
\text { (Lp) } \bigwedge_{i \in I}\left(A_{i} \rightarrow B_{i}\right) \leq E \text { and (Rp) } E \leq C \rightarrow D
$$

Australasian Journal of Logic (18:1) 2020, Article no. 1 
Proposition 5.9. Wherever (Lp) and (Rp) are both instances of axioms, no counterexample to bubbling is provable.

Proof. Cases are numbered by pairs $\langle i, j\rangle$ where $1 \leq i, j \leq 5$, and these numbers stand in for the similarly numbered axiom. So, for instance $\langle 4,5\rangle$ provide premises for (R1) where Lp is an instance of (A4) and Rp an instance of (A5).

First note that merely in virtue of the forms that we need for Lp and Rp, a number of cases are ruled out. For instance, (A4) has a succedent where the main connective is a disjunction, but the only right premises we are concerned with have a conditional as the main connective. Hence $\langle 1,4\rangle$ is of the wrong form as (A4) has succedents of the form $A_{1} \vee B_{1}$ whereas our Rp must have a succedent in which the main connective is a conditional. Another way for a pair of axioms to be ruled out is that they could not form an instance of cut, because $E$ could not have both of the forms required. Hence, $\langle 3,2\rangle$ is ruled out, as then $E$ would have to be both of the form $A_{1} \rightarrow\left(B_{1} \wedge C_{1}\right)$ for (A3) and of the form $A_{2} \wedge B_{2}$ for (A2), and these are incompatible. These two considerations - having the wrong form to produce an instance of (Con), which cases I'll call (WF), or having no cut formula, which I'll call $(\mathrm{NCF})$ - rule out of the following cases:

-WF: $\langle 1,4\rangle,\langle 1,5\rangle,\langle 2,4\rangle,\langle 2,5\rangle,\langle 4,4\rangle$ and $\langle 5, n\rangle$ for any $1 \leq n \leq 5$.

- NCF: $\langle 3,2\rangle,\langle 3,3\rangle,\langle 3,5\rangle,\langle 4,3\rangle,\langle 4,5\rangle$

I'll briefly consider some of the remaining cases, to show that no counterexample can arise (the others are similar to those considered here).

$\langle 1,1\rangle$ In this case, both the left and right premise must be $C \rightarrow D \leq C \rightarrow D$, and we have that $C \leq C$ and $D \leq D$.

$\langle 1,2\rangle$ In this case, the right premise has the form $A^{\prime} \wedge(C \rightarrow D) \leq C \rightarrow D$ and in order for the left premise to have the desired form, it must be that $A^{\prime}$ is a conjunction of conditionals. So the left premise must be of the form $\bigwedge_{i \in I^{\prime}}\left(A_{i} \rightarrow B_{i}\right) \wedge(C \rightarrow D) \leq \bigwedge_{i \in I^{\prime}}\left(A_{i} \rightarrow B_{i}\right) \wedge(C \rightarrow D)$ and the right premise the form $\bigwedge_{i \in I^{\prime}}\left(A_{i} \rightarrow B_{i}\right) \wedge(C \rightarrow D) \leq C \rightarrow D$. Note in this case, again, that $C \leq C$ and $D \leq D$.

$\langle 1,3\rangle$ In order for $C \rightarrow D$ to be the succedent of the right premise, it must be that $D=E \wedge F$, so the right premise of the form $(C \rightarrow E) \wedge(C \rightarrow F) \leq C \rightarrow$ $E \wedge F$. But then, the left premise is still an instance of (A1), in which case note that $C \leq C \wedge C$ and $E \wedge F \leq E \wedge F$.

$\langle 2,1\rangle$ Lp must be of the form $\bigwedge_{i \in I^{\prime}}\left(A_{i} \rightarrow B_{i}\right) \wedge(C \rightarrow D) \leq C \rightarrow D$, and the right premise the correct instance of (A1) for this to fit the desired pattern. In this case, $C \leq C$ and $D \leq D$ suffice again.

$\langle 2,2\rangle$ To fit the required form, we would need for the left premise to be of the form $\bigwedge_{i \in I}\left(A_{i} \rightarrow B_{i}\right) \wedge(C \rightarrow D) \leq \bigwedge_{i \in I^{\prime}}\left(A_{i} \rightarrow B_{i}\right) \wedge(C \rightarrow D)$ and for the right premise to be $\bigwedge_{i \in I^{\prime}}\left(A_{i} \rightarrow B_{i}\right) \wedge(C \rightarrow D) \leq C \rightarrow D$, where $I^{\prime} \subset I$, in which case, we have that $C \leq C$ and $D \leq D$.

Australasian Journal of Logic (18:1) 2020, Article no. 1 
$\langle 2,3\rangle$ In order for the right premise to fit the form, we would need that $D=E \wedge F$, to obtain $(C \rightarrow E) \wedge(C \rightarrow F) \leq C \rightarrow E \wedge F$. In this case, the left premise would have to be of the form $\bigwedge_{i \in I}\left(A_{i} \rightarrow B_{i}\right) \wedge(C \rightarrow E) \wedge(C \rightarrow F) \leq(C \rightarrow$ $E) \wedge(C \rightarrow F)$. But we know that $C \leq C \wedge C$ and $E \wedge F \leq E \wedge F$.

$\langle 3,1\rangle$ Similar to $\langle 1,4\rangle$.

Finally, I present one more suggestive preliminary result.

Proposition 5.10. There can be no counterexample to bubbling resulting from the application of (R1) to a pair Rp, Lp where Rp is an instance of an axiom and Lp is the result of applying (R1) to axioms.

Proof. Suppose that $E \leq C \rightarrow D$ is the result of applying some rule to axioms, and that $\bigwedge_{i \in I}\left(A_{i} \rightarrow B_{i}\right) \leq E$ is an axiom. I'll employ the same naming convention for cases as before, but now the right index of $\langle i, j\rangle$ is the number of an axiom, while the left is that of a rule, and the cases will index the application of (R1) to obtain (Con) by means of the pair of first the axiom of which Rp is an instance, and the rule the application of which Lp is a result.

As before, we can immediately cut down the search space by structural considerations. In particular, we can remove any pair $\langle 5, n\rangle$ for $1 \leq n \leq 6$, as (A5) has a precedent of which $\bigwedge_{i \in I}\left(A_{i} \rightarrow B_{i}\right)$ cannot be an instance. Furthermore, we can ignore any pair whose left element is between 2 and 6 , as all of these rules result in sequents with succedents which cannot have $C \rightarrow D$ as instances. So that leaves eight cases to consider: those $\langle m, n\rangle$ where $1 \leq m \leq 4$ and $n=1$ or $n=4$.

Consider those cases of the form $\langle m, 4\rangle$. For one of these to obtain, it must be that $E$ is of the form $C_{1} \rightarrow D_{1}$, where $C \leq C_{1}$ and $D_{1} \leq D$. But note then that for (R1) to apply to Lp and Rp, it must be that $\bigwedge_{i \in I}\left(A_{i} \rightarrow B_{i}\right) \leq C_{1} \rightarrow D_{1}$ is an axiom, and so we know that $C_{1} \leq \bigwedge A_{j}$ and $\bigwedge B_{j} \leq D_{1}$ for some nonempty $J \subseteq I$. From this it follows that $C \leq \bigwedge_{j \in J} A_{j}$ and $\bigwedge_{j \in J} B_{j} \leq D$, as desired.

Finally, we must consider those cases of the form $\langle m, 1\rangle$, and so we must concern ourselves with axiom-pairs which can be cut together to obtain a sequent of the form $E \leq C \rightarrow D$. Let us call $E \leq F \mathrm{~L}^{R} \mathrm{p}$, and $F \leq C \rightarrow D \mathrm{~L}^{L} \mathrm{p}$. The only axioms which fit the desired form for $\mathrm{L}^{R} \mathrm{p}$ are (A1), (A2), and (A3). I'll consider these cases in turn.

Case: $\mathrm{L}^{R} \mathrm{p}$ is an instance of $(\mathrm{A} 1)$ - i.e. is $C \rightarrow D \leq C \rightarrow D$. Now we must move down a step again and consider subcases according to the axioms of which $E \leq C \rightarrow D$ could be an instance, again showing in all that the cut on Lp and Rp cannot be a counterexample to bubbling.

(1) $\mathrm{L}^{L} \mathrm{p}$ is an instance of (A1). Immediate.

Australasian Journal of Logic (18:1) 2020, Article no. 1 
(2) $\mathrm{L}^{L} \mathrm{p}$ is an instance of (A2). In this case $E$ is of the form $G \wedge(C \rightarrow D)$, in which case $\mathrm{Rp}$ is $\bigwedge_{i \in I}\left(A_{i} \rightarrow B_{i}\right) \leq G \wedge(C \rightarrow D)$. This can only happen when $\mathrm{Rp}$ is an instance of (A1) or (A2). Let us consider the latter. If Rp is an instance of (A2), then $F=A_{k} \rightarrow B_{k}, C=A_{l}$, and $D=B_{l}$ for some $k, l \in I$, but then there's no counterexample.

(3) $\mathrm{L}^{L} \mathrm{p}$ is an instance of (A3). In this case $D=D_{1} \wedge D_{2}$ and $E=\left(C \rightarrow D_{1}\right) \wedge$ $\left(C \rightarrow D_{2}\right)$, but this can only happen if Rp is (A1) or (A2), and the former case is trivial. As for the latter, then $C=A_{k}=A_{l}, D_{1}=B_{k}$, and $D_{2}=B_{l}$ for some $k, l \in I$, and once again we're done.

Case: $L^{R} p$ is an instance of (A2) with the form $G \wedge(C \rightarrow D) \leq C \rightarrow D$. This argument again proceeds by considering what $L^{L} p$ could be, and hence what $R p$ could be, and is similar to previous arguments.

Case: $L^{R} p$ is an instance of (A3) with the form $\left(C \rightarrow D_{1}\right) \wedge\left(C \rightarrow D_{2}\right) \leq C \rightarrow$ $D_{1} \wedge D_{2}$. If this is the case, then $\mathrm{L}^{L} \mathrm{p}$ could be (A1), in which case we're done, or it could be (A2). In this case, Rp can either be (A1) or (A2), and in either case we're done.

It is tedious, but not difficult, to prove the natural partner to Prop 5.10, that no counterexample to bubbling can result from an application of (R1) to Lp (an instance of an axiom) and $\mathrm{Rp}$ (the result of applying some rule to axioms). These results display some nice patterns which could, it seems, be made to coagulate into an inductive argument, but I don't, at the time of writing, see how to do it. So I'll leave off with the conjecture that $\mathbf{B B}_{(\wedge \mathrm{I})}^{+}$bubbles, and this some evidence for it.

It also seems that the extension of $\mathbf{B B}_{(\wedge \mathrm{I})}^{+}$by weak DeMorgan negation, that is a connective just satisfying double negation equivalence and the rule form of contraposition, shouldn't pose substantial further difficulties. So if the bubbling argument can be made to go through, the result of extending the system with such a negation would seem to also succumb to such an argument. Call this system $\mathbf{B B}_{(\wedge \mathrm{I})}$ - this is another seemingly good candidate for composition admission.

5.2. Almost No Logics Bubble. It is known that $\mathbf{B}^{\wedge}$ bubbles, there is reason to think that $\mathbf{B B}_{(\wedge \mathrm{I})}^{+}$bubbles, and to suspect that $\mathbf{B B}_{(\wedge \mathrm{I})}$ does as well. This leads to a natural further question: which other logics bubble? Sadly the title of this section gives away the answer. The following list of axioms (and one rule) have instances (produce conclusions) which counterexemplify bubbling:

$(\vee \mathrm{E})(A \rightarrow C) \wedge(B \rightarrow C) \rightarrow(A \vee B \rightarrow C)$

(Cont) $(A \rightarrow B) \rightarrow(\neg B \rightarrow \neg A)$

(WB) $(A \rightarrow B) \wedge(B \rightarrow C) \rightarrow(A \rightarrow C)$

(WI) $(A \rightarrow B \wedge A) \rightarrow B$

(Cl) $A \rightarrow((A \rightarrow B) \rightarrow B)$

(B) $(A \rightarrow B) \rightarrow((C \rightarrow A) \rightarrow(C \rightarrow B))$

(K) $A \rightarrow(B \rightarrow A)$

Australasian Journal of Logic (18:1) 2020, Article no. 1 
(०I) $A \rightarrow(B \rightarrow(A \circ B))$

$(\mathrm{r} \leftarrow) A \rightarrow(B \rightarrow C) \Leftarrow \Rightarrow B \rightarrow(C \leftarrow A)$

I'll fill in the details for counterexamples fitting a couple of the above axioms, leaving the others for the interested reader. For ( $\vee \mathrm{E})$, note that $\nvdash p \vee q \rightarrow p$, so $\nvdash p \vee q \rightarrow p \wedge q$, though with this axiom we have $\vdash(p \rightarrow r) \wedge(q \rightarrow r) \rightarrow(p \vee q \rightarrow$ $r)$. For $(\circ \mathrm{I})$, note that while $\vdash(p \rightarrow q) \rightarrow(r \rightarrow((p \rightarrow q) \circ r))$, in no reasonable logic do we have $\vdash r \rightarrow p$. As for $(\mathrm{r} \leftarrow)$, the extension of $\mathbf{B B}^{+}$by this rule has (VE) as a theorem.

So while it seems highly likely that $\mathbf{B B}_{(\wedge I)}^{+}$bubbles, it seems that almost no other logics bubble. So this rare, fragile property, while useful for performing surgery on prime theories, is really not to be looked for outside of very minimal extensions to distributive lattice logic.

\section{CONCLUSION}

In this paper I have provided a collection of reasonably simple and natural sufficient conditions for a logic extending $\mathbf{B}^{\wedge}$ to admit channel composition. In addition, I have suggested that the logic $\mathbf{B B}_{(\wedge \mathrm{I})}^{+}$is an obvious candidate for a system admitting channel composites, by means of this argument. Should it turn out that $\mathbf{B B}_{(\wedge \mathrm{I})}^{+}$bubbles, this provides a new, interesting motivation for a potentially interesting logic; one not, to my knowledge, picked out for attention in the past.

The results here suggest a potential unexplored avenue for constructing models of combinatory logics in logics with disjunction. It has long been known that the set of all $\mathbf{B}^{+\top}$ theories does not form a model of combinatory logic. In the past, such models have been constructed using Harrop theories in place of prime theories [14]. Should $\mathbf{B B}_{(\wedge \mathrm{I})}^{+}$prove to bubble, it seems highly likely that one will be able to find, among (a nice subset of) its prime theories, a model of combinatory logic, enriched with union types. This is just a guess, but it strikes me as a potentially interesting avenue for new research on this topic.

\section{REFERENCES}

[1] Alan R. Anderson, Nuel D. Belnap, and J. Michael Dunn. Entailment, The Logic of Relevance and Necessity, Volume II, Princeton University Press (1992).

[2] Henk Barendregt, Mario Coppo, and Mariangiola Dezani-Ciancaglini. A Filter Lambda Model and the Completeness of Type Assignment. Journal of Symbolic Logic 48(4):931-940 (1983). https://doi.org/10.2307/2273659

[3] Henk Barendregt, Wil Dekkers, and Richard Statman. Lambda Calculus with Types. Cambridge University Press (2013).

[4] Jon Barwise. Constraints, Channels, and the Flow of Information, Situation Theory and Its Applications 3, ed. Aczel, Israel, Katagiri, and Peters. CLSI Publications, pp.3-27 (1993).

[5] Jon Barwise, Dov Gabbay, and Chrysafis Hartonas. Information Flow and the Lambek Calculus. In Logic, Language, and Computation: 1994 Proceedings ed. Jerry Seligman and Dag Westerståhl. CSLI Publications (1995).

[6] Jon Barwise, Dov Gabbay, and Chrysafis Hartonas. On the Logic of Information Flow. Logic Journal of the IGPL 3(1):7-49 (1995). https: //doi .org/10.1093/jigpal/3.1.7

[7] Jon Barwise and John Perry. Situations and Attitudes. MIT Press (1983).

Australasian Journal of Logic (18:1) 2020, Article no. 1 
[8] Jon Barwise and John Perry. Shifting Situations and Shaken Attitudes. Linguistics and Philosophy 8:105-161 (1985). https://doi .org/10.1007/BF00653379

[9] Nuel D. Belnap A Formal Analysis of Entailment. Technical Report no. 7, Prepared under Contract SAR/Nonr-609(16) for the Office of Naval Research, Yale University (1960).

[10] Nuel D. Belnap. A Useful Four-Valued Logic. Modern Uses of Multiple-Valued Logic ed. J. Michael Dunn and George Epstein, pp 5-37 (1977). link.springer.com/ chapter/10.1007/978-94-010-1161-7_2

[11] Katalin Bimbó and J. Michael Dunn. The Emergence of Set-Theoretical Semantics for Relevance Logics around 1970. Proceedings of the Third Workshop, Special Issue of IfCoLog Journal of Logics and Their Applications ed. Katalin Bimbó and J. Michael Dunn, 4(3):557-589 (2017). collegepublications.co.uk/downloads/ifcolog00012.pdf

[12] Ross T. Brady. Relevant Logics and Their Rivals, Volume II: A Continuation of the Work of Richard Sylvan, Robert Meyer, Val Plumwood and Ross Brady. (Edited by Ross Brady with contributions by Martin Bunder, André Fuhrmann, Andréa Loparić, Edwin Mares, Chris Mortensen and Alasdair Urquhart.) Ashgate (2003).

[13] Ross T. Brady. Metavaluations. Bulletin of Symbolic Logic 23(3):296-323 (2017). https://doi.org/10.1017/bsl.2017.29

[14] Mariangiola Dezani-Ciancaglini, Robert K. Meyer, and Yoko Motohama. The Semantics of Entailment Omega. Notre Dame Journal of Formal Logic 43(3):129-145 (2002). https://projecteuclid.org/euclid.ndjfl/1074290712\#abstract

[15] J. Michael Dunn and Gary Hardegree. Algebraic Methods in Philosophical Logic Oxford University Press (2005).

[16] J. Michael Dunn and Robert K. Meyer. Combinators and Structurally Free Logic. Logic Journal of the IGPL 5(4):505-537 (1997). https://doi .org/10.1093/jigpal/5.4.505

[17] Lou Goble. Neighbourhoods for Entailment. Journal of Philosophical Logic 32:483-529 (2003). doi.org/10.1023/A:1025638012192

[18] Ronald Harrop. Concerning Formulas of Types $A \rightarrow B \vee C, A \rightarrow(E x) B(x)$ in Intuitionistic Formal Systems. Journal of Symbolic Logic 25(1):27-32 (1960). doi .org/10.2307/2964334

[19] Lloyd Humberstone. The Connectives MIT Press (2011).

[20] Peter Lavers. Generating Intensional Logics. M.A. Thesis, University of Adelaide (1985).

[21] Peter Lavers. Generalising Tautological Entailment. Logique et Analyse 146:367-377 (1994). https://www.jstor.org/stable/44084401

[22] Edwin Mares. Information Flow and the Theory of Information. Synthese 109:345-360 (1996). https://doi.org/10.1007/BF00413865

[23] Edwin Mares. Relevant Logic: A Philosophical Interpretation. Cambridge University Press (2004).

[24] Robert K. Meyer. Metacompleteness. Notre Dame Journal of Formal Logic 17(4):501-516 (1976). https://projecteuclid.org/euclid.ndjfl/1093887722

[25] Robert K. Meyer. A Better Bubbling Lemma. Electronic Notes in Theoretical Computer Science 171:77-84 (2007). https: //doi . org/10.1016/j .entcs . 2006.12.038

[26] Robert K. Meyer, Martin W. Bunder, and Lawrence Powers. Implementing the 'Fool's Model' of Combinatory Logic. Journal of Automated Reasoning 7:597-630 (1991). https://doi.org/10.1007/BF01880331

[27] Robert K. Meyer and Richard Routley. Algebraic Analysis of Entailment I Logique et Analyse 15(59-60) (1972). virthost.vub.ac.be /lnaweb/ojs/index.php/LogiqueEtAnalyse/article/view/652

[28] Koushik Pal and Robert K. Meyer. Basic Relevant Theories for Combinators at Levels I and II. Australasian Journal of Logic 3:1-22 (2005). doi . org/10. 26686/aj1 . v3i0.1770

[29] Greg Restall. Information Flow and Relevant Logics. In Logic, Language, and Computation: 1994 Proceedings ed. Jerry Seligman and Dag Westerståhl.CSLI Publications, pp. 463-477 (1995).

[30] Greg Restall. An Introduction to Substructural Logics. Routledge, London (2000).

[31] Richard Routley and Robert K. Meyer. The Semantics of entailment. In Truth, Syntax and Modality ed. Hugues Leblanc North Holland, pp. 194-243 (1972).

Australasian Journal of Logic (18:1) 2020, Article no. 1 
[32] Richard Routley and Robert K. Meyer. Towards a General Semantical Theory of Implication and Conditionals. I. Systems with Normal Conjunctions and Disjunctions and Aberrant and Normal Negations. Reports on Mathematical Logic 4:67-89 (1975).

[33] Richard Routley and Robert K. Meyer. Towards a General Semantical Theory of Implication and Conditions. II. Improved Negation Theory and Propositional Identity. Reports on Mathematical Logic 9:47-62 (1976).

[34] Richard Routley and Robert K. Meyer. Every Sentential Logic has a Two-Valued Worlds Semantics. Logique et Analyse 19(74/76):345-365 (1976). jstor .org/stable/44084526

[35] Richard Routley, Robert K. Meyer, Valerie Plumwood. Ross T. Brady. Relevant Logics and Their Rivals 1. Ridgeview (1982).

[36] Richard Routley and Valerie Routley. The Semantics of First Degree Entailment. Noûs 6(4):335359 (1972). jstor .org/stable/pdf/2214309.pdf

[37] Sebastian Sequoiah-Grayson. Epistemic Closure and Commutative, Nonassociative Residuated Structures. Synthese 190(1):113-128 (2013). doi .org/10.1007/s11229-010-9834-z

[38] Sebastian Sequoiah-Grayson. Epistemic Relevance and Epistemic Actions. J. Michael Dunn on Information Based Logics, ed. Katalin Bimbó, Springer, p.133-146 (2016). doi.org/10.1007/978-3-319-29300-4_8

[39] Andrew Tedder. Channel Composition and Ternary Relation Semantics. Proceedings of the Third Workshop, Special Issue of IfCoLog Journal of Logics and Their Applications ed. Katalin Bimbó and J. Michael Dunn, 4(3):731-753 (2017). collegepublications.co.uk/downloads/ifcolog00012.pdf

[40] Alasdair Urquhart. Semantics for Relevant Logics. Journal of Symbolic Logic 37(1):159-169 (1972). doi.org/10.2307/2272559

Australasian Journal of Logic (18:1) 2020, Article no. 1 